Article

\title{
PVDF Sensor Stimulated by Infrared Radiation for Temperature Monitoring in Microfluidic Devices
}

\author{
Salvatore A. Pullano ${ }^{1,2, *}$, Ifana Mahbub ${ }^{2}$, Syed K. Islam ${ }^{2}$ and Antonino S. Fiorillo ${ }^{1}$ \\ 1 Department of Health Sciences, University Magna Græcia of Catanzaro, Viale Europa, \\ 88100 Catanzaro, Italy; nino@unicz.it \\ 2 Department of Electrical Engineering and Computer Science, University of Tennessee, 1520 Middle Drive, \\ Knoxville, TN 37996, USA; imahbub@vols.utk.edu (I.M.); sislam@utk.edu (S.K.I.) \\ * Correspondence: pullano@unicz.it; Tel.: +39-961-369-4307
}

Academic Editors: Guillermo Villanueva and Tom Larsen

Received: 5 January 2017; Accepted: 11 April 2017; Published: 13 April 2017

\begin{abstract}
This paper presents a ferroelectric polymer-based temperature sensor designed for microfluidic devices. The integration of the sensor into a system-on-a-chip platform facilitates quick monitoring of localized temperature of a biological fluid, avoiding errors in the evaluation of thermal evolution of the fluid during analysis. The contact temperature sensor is fabricated by combining a thin pyroelectric film together with an infrared source, which stimulates the active element located on the top of the microfluidic channel. An experimental setup was assembled to validate the analytical model and to characterize the response rate of the device. The evaluation procedure and the operating range of the temperature also make this device suitable for applications where the localized temperature monitoring of biological samples is necessary. Additionally, ease of integration with standard microfluidic devices makes the proposed sensor an attractive option for in situ analysis of biological fluids.
\end{abstract}

Keywords: pyroelectric devices; temperature sensor; system-on-a-chip; ferroelectric materials; thin film sensors

\section{Introduction}

One of the most important aims of the development of a biomedical system-on-a-chip (SoC) is to achieve successful integration of sensors and actuators into a low-cost device which will provide a wide range of functionalities required for most applications [1]. In recent literature, several devices implementing on SoC platform have been reported for detection of genetic variation, integrated polymerase chain reaction, and electrophoretic analysis [2-4]. These devices usually have low thermal inertia-a characteristic that allows for rapid changes in the temperature of the biological samples. This phenomenon occurs frequently and requires innovative technological solutions for its rapid, localized, and accurate assessment. It is particularly relevant in the case of fast temperature changes (in the millisecond time scale) where it is known to be one of the primary causes of errors [5-8]. Various transducer technologies have been employed for in situ temperature measurement such as thermocouples, thermistors, and thermochromic liquid crystals, but there is still an increasing demand for low-cost contact sensors in micro-scale applications [9-13]. The performance of a SoC device can be improved by incorporating integrated temperature transducer with dimensions of few $\mu \mathrm{m}^{2}$ of area and featuring fast response, high accuracy, easy integration, low power consumption, and improved reliability. Among various available transducer technologies, the polymer sensor is one of the fastest growing technologies for commercialization and has maturated for practical applications from a long list of candidates. Ferroelectric polymers such as polyvinylidene fluoride (PVDF) is a polarized fluoropolymer that, in its polar form ( $\beta$-phase), demonstrates strong and stable 
piezoelectric and pyroelectric activities. Thermal transducers employing a thin pyroelectric film of PVDF can be designed to provide fast response time. In addition, the pyroelectric voltage coefficient of PVDF is about an order of magnitude larger than that of other pyroelectric materials such as lead zirconate titanate $(\mathrm{PZT})$ or barium titanate $\left(\mathrm{BaTiO}_{3}\right)$ [14].

The pyroelectric transducer provides an electrical response due to the change in its temperature and therefore typically requires an adjunctive reference sensor to provide information about the target temperature. However, recent literature reported efforts on the development of high performances pyroelectric temperature sensors that avoid supplementary components representing a promising technology, particularly for medical applications [15-17]. In previous work, PVDF-based pyroelectric sensors were investigated for monitoring of fast temperature variations in microfluidic devices. Due to the dynamic nature of the PVDF transducer, the device only provided information on the temperature gradient but not the initial and final temperature values [18-20]. Moreover, the respectable degree of compatibility of PVDF with most of the polymeric substrates used for fabrication of disposable devices (e.g., polymethylmethacrylate, etc.) makes it suitable for integrated sensor applications [21,22].

In this paper, a modified pyroelectric transducer is investigated for overcoming previous limitations in the evaluation of the absolute temperature inside a microfluidic channel. Moreover, a thermal model based on the analysis of heat transfer mechanism is developed for the characterization of the transducer. The pyroelectric contact thermometer is composed of a thin film of PVDF coupled with a microfluidic device and includes an infrared (IR) light source and a charge amplifier that generates a suitable voltage signal in response to the electrical charge [20,23-25]. As a consequence of the modified design of the transducer, the use of mechanical components (such as shutters) and adjunctive reference sensor (e.g., thermocouple) are eliminated in the proposed configuration. Simulations of the developed thermal model were compared with the experimental results for the validation of the proposed sensor. The working bandwidth of the system $(0.8$ to $49.7 \mathrm{~Hz})$ can be adjusted for specific applications by modifying the geometry of the transducer and the front-end circuitry. Comparison of the proposed sensor with other temperature sensors reported in literature shows a faster response time along with a smaller footprint thereby making the proposed sensor suitable for SoC applications.

\section{Pyroelectric Sensor Design}

\subsection{Device Fabrication}

The device was fabricated using a uniaxially stretched $28 \mu \mathrm{m}$ thick sheet of PVDF (Measurement Specialties, Hampton, VA, USA) which is a ferroelectric polymer synthesized in polycrystalline form and exhibits a strong and stable pyroelectric effect with the direction of the polarization axis being perpendicular to both surfaces. Attenuated total reflection Fourier transform infrared (ATR-FTIR) spectroscopy has been used to characterize molecular conformation of PVDF. Among different crystalline structures of PVDF, $\beta$-phase is the most interesting for technological purpose due to its stronger piezoelectric and pyroelectric properties [26]. In particular, higher $\beta$-phase conformation film provides higher yield within the thin film of PVDF. Figure 1a shows a representative FTIR-ATR spectrum of PVDF film in which peaks around 1278 and $1238 \mathrm{~cm}^{-1}$ highlight the film containing both $\beta$-phase and $\gamma$-phase while higher peaks at about $840 \mathrm{~cm}^{-1}$ indicate a PVDF film with a higher content of $\beta$-phase crystalline structures. Moreover, analysis performed along the sample showed a very good degree of uniformity, which thus reduced differences in pyroelectric response all along the film. SEM analysis of Figure $1 \mathrm{~b}$ underlines a good surface quality in terms of roughness and density, which leads to a good quality PVDF film for thermo-electrical application [27]. 


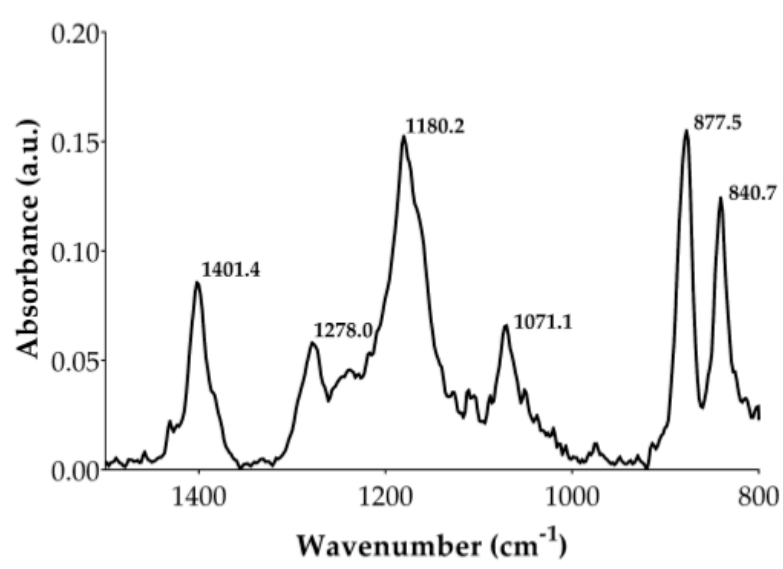

a

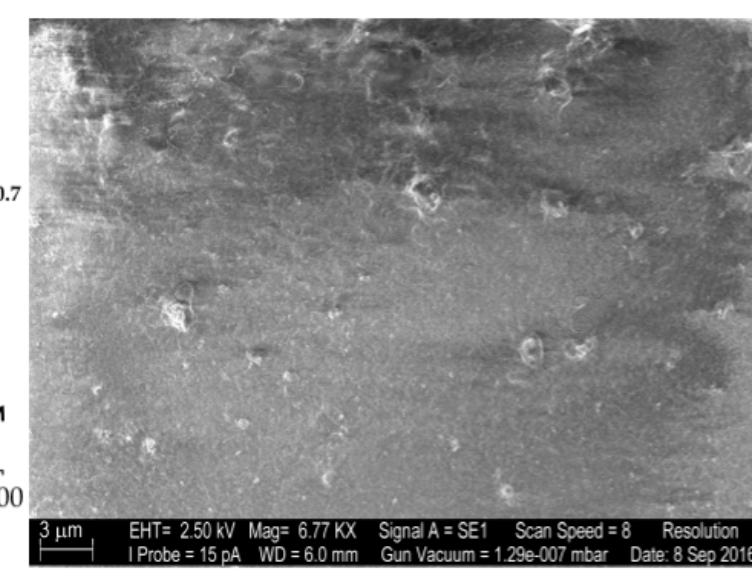

$\mathrm{b}$

Figure 1. Polyvinylidene fluoride (PVDF) characterization obtained by (a) Fourier transform infrared spectroscopy and (b) scanning electron microscopy.

Sputtered gold (Emitech K650X, EM Technologies Ltd., Ashford, UK) was used to obtain $300 \mathrm{~nm}$-thick electrodes on both faces of the sheet at a temperature sufficiently lower than the transition temperature to avoid depolarization. Gold metallization layers were fabricated to be overlapped only in the area over the microchannel (i.e., active transducer area), which in the actual arrangement was measured to be $2 \times 0.5 \mathrm{~mm}^{2}$. The metallization outside the microchannel was not overlapped and just provided electrical pathways for signal acquisition and analysis. A thin black layer of graphite was sputtered onto the active area and an infrared LED source was then fixed in contact with the transducer. Each layer added onto PVDF sheet creates an additional heat path that is undesirable since it reduces the efficiency of delivering heat to/from the pyroelectric element limiting the sensitivity and the resolution. In order to reduce the additional heat paths, the gold metallization and the graphite layer (about $500 \mathrm{~nm}$ in thickness) were designed to be thinner compared to the PVDF film. Moreover, the graphite layer allows most of the infrared energy transmitted by the IR source to be absorbed and not reflected by the metallic layer. The transducer was permanently fixed on the surface of the microfluidic chip through a PMMA interlayer that electrically insulated it, while the external contact was realized via ultrasound soldered gold conductors. The interlayer was realized with a solution of PMMA (Röhm, Milano, Italy) and Anisole 99\% (Carlo Erba, Milano, Italy) that was first spin-coated (1 $\mu \mathrm{m}$ in thickness) onto the lower PVDF surface as shown in Figure 2 [19]. Anisole was used as a solvent for PMMA with reduced health-related issues.

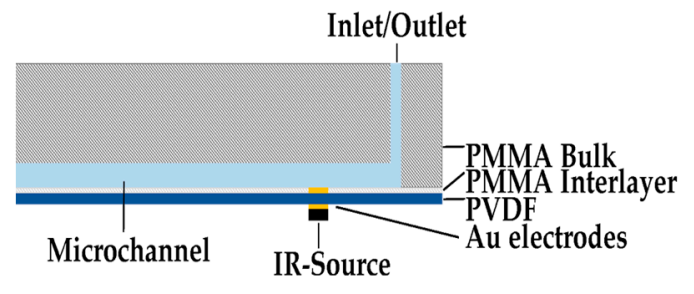

(a)

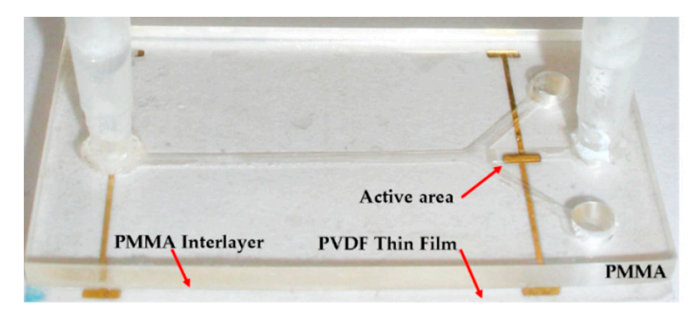

(b)

Figure 2. (a) Cross-section and (b) picture of the microfluidic device containing the micro-milled PMMA bulk and the PVDF thin film on which the gold metallizations are deposited to serve as active element and electrical connections to the read-out electronic circuit.

The PMMA bulk hosts the holes for inlet and outlet connections. PMMA and PVDF layers were sealed by means of a liquid PMMA interlayer spun onto the PVDF and then pressed toward the PMMA bulk. 


\subsection{Pyroelectric Charge Generation}

The pyroelectric effect is the manifestation of the temperature dependence of the spontaneous polarization in certain anisotropic solids. When the temperature $\theta$ of a pyroelectric material is changed, a voltage is produced across the sensor [28]. Assuming the sample heating rate to be significantly smaller than the thermal diffusivity time, the sample can be considered to be uniformly heated and thus the generated voltage $d V_{p}$ or equivalently the charge $d Q_{p}$, is described by,

$$
d V_{p}=p_{V} h d \theta, d Q_{p}=p_{Q} A d \theta
$$

where $p_{V}$ and $p_{Q}$ are the voltage- and the charge-pyroelectric coefficients and $h$ and A are the thickness and the area of the material, respectively. Pyroelectric coefficients are related to each other by the ratio $p_{Q} / p_{V}$ which corresponds to the permittivity of the material, $\varepsilon$. The response is achieved when the surface of the material is perpendicular to the direction of polarization [29]. A pyroelectric response is stimulated on a pyroelectric thin film, metallized on both surfaces, with an IR impulse to impose a temperature difference between the top absorbing layer and the lower face, $\theta_{P}(t)=\theta_{U}(t)-\theta_{L}$ (taken as reference during IR stimulation) [30]. If the thermal wave that propagates through the outer absorbing metallic layer is not taken into account then considering the thermal diffusivity of the metal (e.g., gold) to be much higher than that of the pyroelectric material and at the same time its thickness to be very small, $\theta_{P}(t)$ following an infrared impulse is dictated by,

$$
C_{t h} \frac{d \theta_{p}(t)}{d t}+\frac{1}{R_{t h}} \theta_{p}(t)=\alpha P(t)
$$

where $C_{t h}$ and $R_{t h}$ are the thermal capacitance and resistance of the PVDF film, respectively, $\alpha$ is the absorption coefficient of the outer metallic layer on which the radiation is incident and $P(t)$ is the radiation power $[30,31]$. The response time of the pyroelectric transducer is related to its thermal time constant, $\tau_{t h}=C_{t h} \cdot R_{t h}$. By using Laplace transform, the convolution theorem, and inverse Laplace transform, we can rewrite Equation (2) as,

$$
\theta_{p}(t)=\frac{\alpha \tau_{t h} U}{C_{t h}}\left(e^{\frac{T_{I R}}{\tau_{t h}}}-1\right) e^{\frac{-t}{\tau_{t h}}}
$$

where the term $U$ represents the energy of the infrared source. Equation (3) highlights that $\theta_{P}(t)$ rises almost instantly to its maximum value following the IR stimulation $\left(\theta_{P}(0)\right)$. Afterward, it decreases within a time defined by the thermal time constant, $\tau_{t h}$. By using first order Taylor series expansion and considering the step duration of the IR source, $T_{I R}<<\tau_{t h}$, Equation (3) becomes,

$$
\theta_{p}(t)=\frac{\alpha \tau_{t h} U}{C_{t h}}\left(e^{\frac{T_{I R}}{\tau_{t h}}}-1\right)\left(1-\frac{t}{\tau_{t h}}\right)
$$

Temperature evolution, $\theta_{P}(t)$, following a given IR laser impulse, is reported in Figure 3 using the same geometrical dimensions (i.e., length width and thickness) and thermal properties (i.e., specific heat, thermal conductivity) of the fabricated device and following Equation (4). $C_{t h}$ and $R_{t h}$ mainly affect the return to thermal equilibrium of the pyroelectric film. In particular, higher the value of $\tau_{\text {th }}$ the slower is the return to the thermal equilibrium.

Stimulating the pyroelectric transducer with an IR source impulse with $T_{I R}<<\tau_{t h}$, a current is thus generated, which decreases exponentially following the thermal time constant of the transducer. Considerations can be made regarding the generation of a pyroelectric charge using Equation (4) under the condition $T_{I R}<<\tau_{\text {th }}$ and neglecting the quadratic terms, which according to Equation (1) becomes:

$$
Q_{p}(t) \cong \frac{p A \alpha U}{C_{t h}} t
$$


Therefore, based on the above-mentioned assumption, the charge generated is a linear function of time.

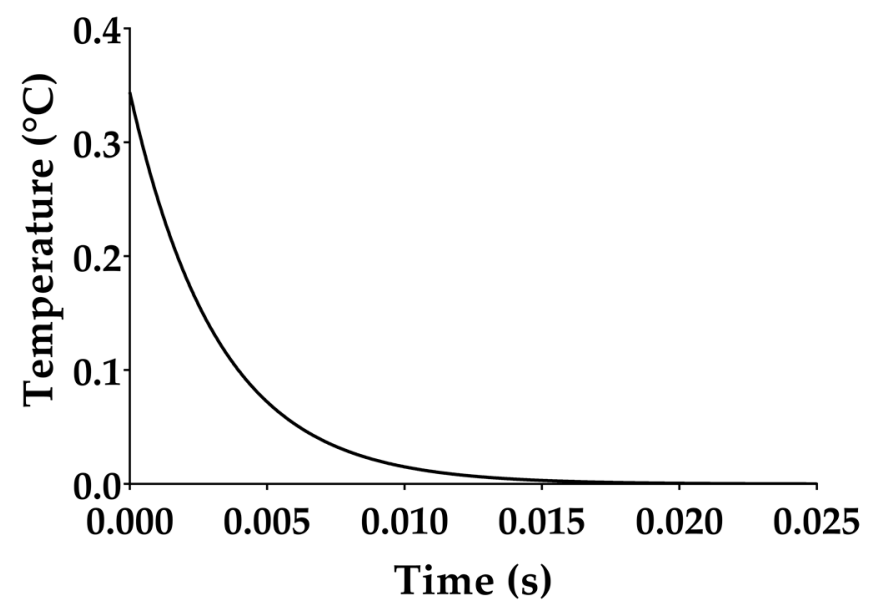

Figure 3. Analytical temperature difference $\theta_{p}(t)$ between the top absorbing layer, $T_{U}(t)$, and the lower face, $T_{L}$, following a step-shaped infrared (IR) radiation source of $50 \mathrm{~mW}$ with a time duration $T_{I R}=0.3 \mathrm{~ms}$ and considering the parameters reported in Table 1. By considering similar radiation impulse, according to Equation (4), the higher the thermal time constant, the longer the return to thermal equilibrium $\left(\theta_{U}(t)=\theta_{L}\right)$.

\subsection{Charge Amplifier}

A charge amplifier implemented as a current integrator integrates a pulse of current and produces an output voltage $V_{o}$ proportional to the pyroelectric charge through the factor $1 / C_{f}$. Feedback capacitance is designed to make the transfer process independent of the transducer and any other stray capacitances, while feedback resistance provides a current path for the feedback capacitor to be reset [32,33]. It is characterized by a high input and low output impedance. The input impedance of the charge amplifier is composed of the input impedance of the operational amplifier as well as the inherent PVDF capacitance $C_{0}$ and a leakage resistance $R_{0}$. The gain of the charge amplifier is also influenced by the charge transfer from $C_{0}$ to $C_{f}[34,35]$. Figure 4 shows the schematic diagram of the charge amplifier with a feedback capacitance $\left(C_{f}=1 \mathrm{nF}\right)$ and resistance $\left(R_{f}=100 \mathrm{M} \Omega\right)$ which is used to convert the temperature-dependent charge collected on the metallized surfaces into a proportional voltage.

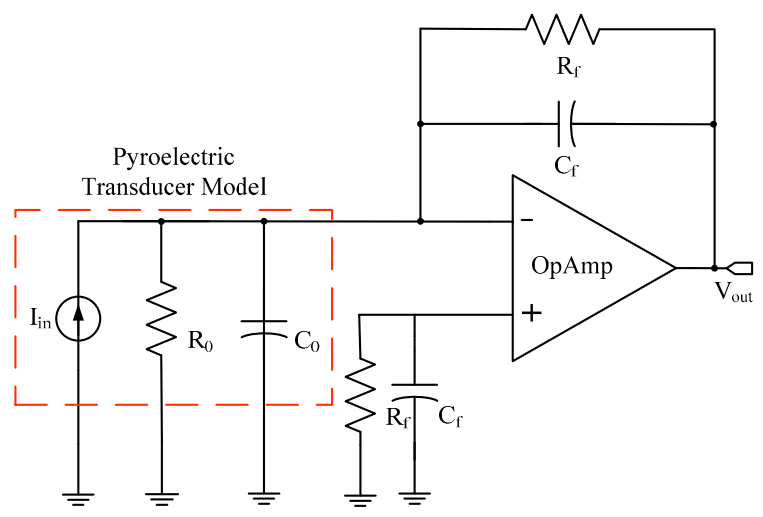

Figure 4. Schematic diagram of the designed charge amplifier.

OPA124 op-amp is used for implementation of the front-end charge amplifier, which provides a high gain-bandwidth-product $(\mathrm{GBW}=1.5 \mathrm{MHz})$, low bias current $\left(I_{b}=1 \mathrm{pA}\right.$ max. $)$, high slew-rate 
$(\mathrm{SR}=1.6 \mathrm{~V} / \mu \mathrm{s})$, and high DC open loop gain $\left(A_{v o l}=125 \mathrm{~dB}\right)$. Moreover, its high input impedance $\left(Z_{\text {in }}=10^{13} \Omega|| 1 \mathrm{pF}\right)$ avoids bleed-off of the charge on the feedback capacitor and the low bias current prevents the feedback capacitor from charging and discharging at excessive rates [19]. $C_{0}$ and $R_{0}$ have been evaluated at low frequencies using a Keithley 4200-SCS source meter resulting in $C_{0}=5.01 \pm 0.09 \mathrm{pF}$ and $R_{0}=213.8 \pm 53.8 \mathrm{G} \Omega$ at $1.32 \mathrm{~Hz}$. The electrical time constant $\tau_{e}=R_{\text {in }} \cdot C_{\text {in }}$ depends on the equivalent input resistance $R_{i n}=R_{f} /(1+\beta A)|| R_{0}$ shunted by the input equivalent capacitance $C_{\text {in }}=C_{f}(1+\beta A)|| C_{0}$, where $\beta=-1 / Z_{f}$ is the feedback factor and $A=-A_{\text {vol }} Z_{f}$ is the open loop gain (i.e., $\tau_{e}=100 \mathrm{~ms}$ ) [20]. $C_{f}$ and $R_{f}$ on the non-inverting input are used to balance the source impedances (both resistive and reactive) in order to minimize input bias current errors and output noise together with shielding through input guard [34]. Both the thermal and the electrical time constants define a frequency band of the transducer (in the actual configuration is $0.8-49.7 \mathrm{~Hz}$ ). By neglecting the output resistance and assuming an ideally infinite input resistance of the operational amplifier, Equation (6) can be written as,

$$
V_{o}=-\frac{Q_{p}}{C_{f}} e^{-t / \tau_{e}}
$$

Here, the signal amplitude decreases following the time constant, $\tau_{e}$ and the maximum value of the response is a measure of the temperature variation.

\subsection{Heat Transfer Process}

Recently, there has been a great deal of interest in the use of pyroelectric effect for making thermal measurements and detecting electromagnetic radiations [36-38]. Low-temperature differential measurements, such as the evaluation of specific heat require high sensitivity and minimum joule heating, are readily provided by the pyroelectric devices $[39,40]$. There are different ways to characterize a thermometer, especially in the initial evaluation phase, by developing a correlation between the physical properties of the transducer with variations in temperature or by the evaluation of a thermal property of the material itself [41]. From the thermal point of view (see Figure 5), a pyroelectric transducer mounted on a microfluidic chip is governed by the following mechanisms: IR absorption, thermal radiation and thermal conduction. The absorption of the IR radiation on the outer layer generates heat that results in the rise of the temperature and a net heat flow is described as,

$$
\Delta W_{I R}=A \alpha I \Delta t
$$

where $\mathrm{A}$ is the surface, $\alpha$ is the surface absorption coefficient and $I$ is the irradiance. The heat transfer mechanisms of PVDF transducer involving thermal radiation and the thermal conduction are described using the following equations, respectively:

$$
\begin{gathered}
\Delta W_{T R}=A \delta \sigma \theta^{4} \Delta t \\
\Delta W_{\text {Cond }}=\frac{1}{R_{t h}} \theta_{p}(t) \Delta t
\end{gathered}
$$

where $\delta$ is the surface emissivity and $\sigma$ is the Stefan-Boltzmann constant. The overall thermal effect, which gives rise to temperature variations, is described as follows:

$$
\Delta W_{t o t}=A \alpha I \Delta t-A \delta \sigma \theta^{4} \Delta t-\frac{1}{R_{t h}} \theta_{p}(t) \Delta t=C_{t h} \theta_{p}(t)
$$

Therefore, the response to an infrared pulse is dependent on the absolute temperature according to Equation (10). The IR absorption term is expected to be constant due to the intensity of the IR source and the thermal radiation term depends on $\theta^{4}$. The term that takes into account the thermal conduction is expected to be constant and small if compared with the previous terms, as the total radiant flux is 
maintained at a low value (very small temperature change on the pyroelectric element due to IR source if compared with the target temperature). Therefore, increasing the target temperature of the device results in a heat flow dominated by thermal radiation mechanism (i.e., $\theta^{4}$ ). By taking into account the contribution of the charge amplifier, Equation (10) can be rewritten as,

$$
\left(-\frac{V_{o}}{p A} C_{f}\right) C_{t h}=A \alpha I \Delta t-A \delta \sigma \theta^{4} \Delta t-\frac{1}{R_{t h}} \theta_{p}(t) \Delta t=C_{t h} \theta_{p}(t)
$$

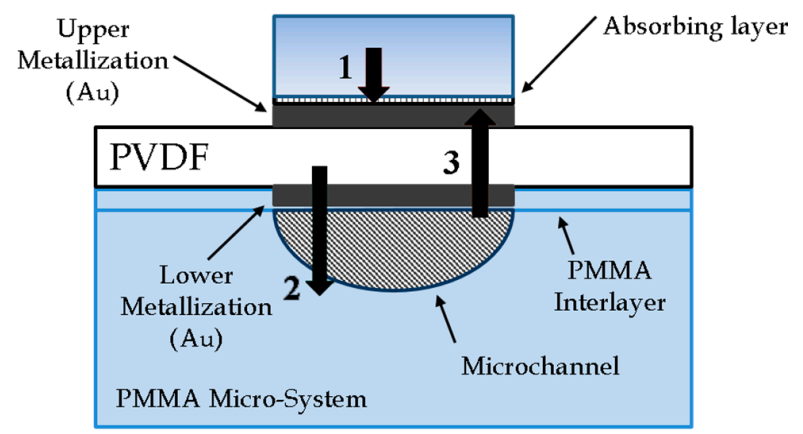

Figure 5. Cross section of the device highlighting with the black arrows the heat transfer mechanisms involving (1) infrared thermal absorption, (2) thermal radiation, and (3) thermal conduction.

For a given $\Delta t$, Equation (11) relates the output voltage of the pyroelectric sensor to its temperature. Figure 6 shows the analytical temperature dependence of $V_{o}$ by plotting Equation (11) using the parameters reported in Table 1 and by varying the temperature from $0{ }^{\circ} \mathrm{C}$ to $65^{\circ} \mathrm{C}$.

Table 1. Device parameters of the pyroelectric sensor.

\begin{tabular}{ccc}
\hline \multicolumn{3}{c}{ Value } \\
\hline Active surface $[A]$ & $10^{-6}$ & {$\left[\mathrm{~m}^{2}\right]$} \\
Thermal capacitance $\left[C_{t h}\right]$ & $32 \times 10^{-6}$ & {$\left[\mathrm{~J} \cdot \mathrm{K}^{-1}\right]$} \\
Absorption coefficient $[\alpha]$ & 0.7 & \\
Irradiance $[I]$ & $10^{-3}$ & {$\left[\mathrm{~W} \cdot \mathrm{m}^{-2}\right]$} \\
Surface emissivity $[\delta]$ & 0.7 & \\
Stefan-Boltzmann constant $[\sigma]$ & $5.67 \times 10^{-8}$ & {$\left[\mathrm{~W} \cdot \mathrm{m}^{-2} \cdot \mathrm{K}^{-4}\right]$} \\
PVDF specific heat $\left[c_{p}\right]$ & $1.4 \times 10^{3}$ & {$\left[\mathrm{~J} \cdot \mathrm{kg}^{-1} \cdot \mathrm{K}^{-1}\right]$} \\
PVDF thermal conductivity $\left[R_{t h}{ }^{-1}\right]$ & 0.2 & {$\left[\mathrm{~W} \cdot \mathrm{m}^{-1} \cdot \mathrm{K}^{-1}\right]$} \\
\hline
\end{tabular}

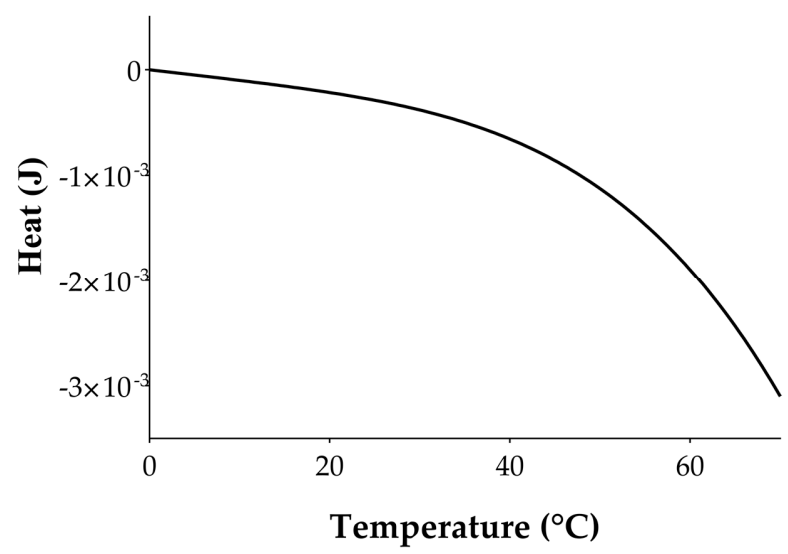

Figure 6. Temperature dependence of the response of the pyroelectric sensor to the heat transfer mechanism. For a given time of evaluation, $t_{0}$ provides the relationship between the output voltage $V\left(t_{0}\right)$ and the temperature of the microchannel. 
Therefore, considering Equation (11), the microchannel temperature can be evaluated exploiting the characteristic of the pyroelectric element that is faster compared to the traditional integrated sensors because there is no latency time for the thermalization. In fact, pyroelectric response takes place instantaneously if thermal equilibrium is perturbed (inducing a difference in temperature $\left.\theta_{p}(t)\right)$, while other temperature sensors (e.g., thermocouples, thermistors) need the establishment of thermal equilibrium prior to the evaluation of the temperature. Concerning the dimension of the transducer, a large-sized transducer leads to larger thermal capacitance caused by the volume of the larger transducer. Consequently, the transducer is characterized by higher thermal inertia and a larger amount of heat will be required to bring the transducer to the target temperature. Thus, the lower the value of the thermal capacitance, the less significant the change in temperature of the target object.

\section{Discussion}

Two experimental setups were developed in order to carry out the measurements required for the necessary validation of the analytical model. The first was the adjustment of the sensor in an environmental test chamber maintained at a constant temperature (Delta Design, Delta 9023). In this case, the obtainable temperature accuracy is $\pm 1.0^{\circ} \mathrm{C}$ whilst the temperature deviation after stabilization is $\pm 0.1^{\circ} \mathrm{C}$. The second setup consisted of a hot plate (Torrey Pines Scientific ${ }^{\circledR}, \mathrm{HS} 40$, Carlsbad, CA, USA) that is thermally controlled by a Platinum RTD and is characterized by an accuracy of $\pm 1 \%$. Similar results were obtained by both setups, although the environmental test chamber yielded results that were less prone to electromagnetic noise (working as Faraday cage-like) and were more reliable for maintaining the channel temperature at the required level. The pyroelectric response was acquired by the front-end charge amplifier and then recorded by an Agilent MSO X3054A oscilloscope. A light-emitting diode operating at $850 \mathrm{~nm}$ wavelength was used as the infrared source, while a signal generator was used for the infrared impulse modulation (Agilent 33220A, Santa Clara, CA, USA) and was set at a suitable frequency, duty cycle, and amplitude. The IR diode was a high-power LED with an emission angle of $\pm 3^{\circ}$ and a maximum radiant flux of $50 \mathrm{~mW}$ which irradiates an area of $10^{-6} \mathrm{~m}^{2}$. The instruments were warmed up for $2 \mathrm{~h}$ prior to the experiment in order to prevent any temperature variation that might cause a drift in the measurement results. Experimental data obtained for the temperature range of $25-65^{\circ} \mathrm{C}$ highlights the fact that the behavior of the sensor at each temperature is governed by both the thermal (rise phase) and the electrical (fall phase) characteristics of the sensor response. Figure 7 shows pyroelectric responses recorded at the charge amplifier output choosing $T_{I R}=0.3 \mathrm{~ms}$ (with $T_{I R}<\tau_{t h}$ ). The results indicate that $d V_{o} / d t$ in the time frame considered decreases with the increase in the temperature.

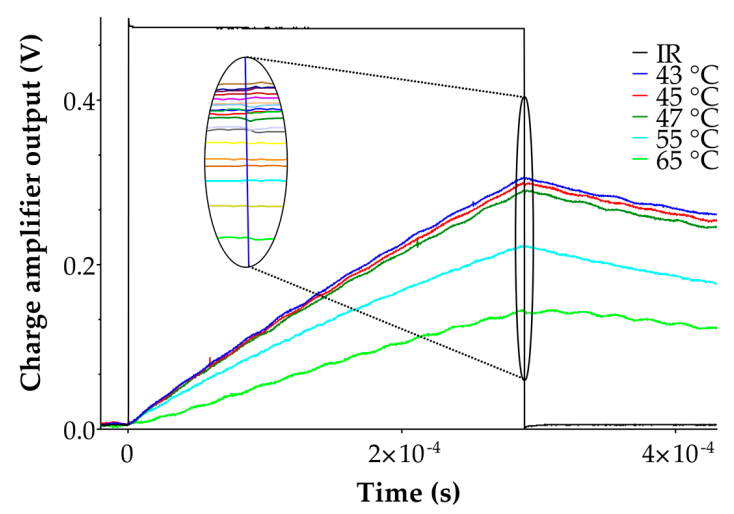

Figure 7. Experimental data obtained at five different target temperatures using the heating chamber. The square signal in black represents the signal generated for the IR-LED stimulation. The colored signals represent the output of the sensor at different target temperature. The slope in the time interval $\left[0, T_{I R}\right]$, varies depending on the microchannel temperature (at higher temperatures the response slope decrease). All the responses obtained in the range $25-65^{\circ} \mathrm{C}$ are highlighted and the maximum points of the responses at $t=T_{I R}$ decrease according to Equation (11). 
For a given microchannel temperature, the sensor was stimulated with IR radiation, and the voltage $\left(V_{o}\right)$ at $t=0.3 \mathrm{~ms}$ was evaluated and recorded. Figure 8 displays all the recorded values at the charge amplifier output at the time $t=0.3 \mathrm{~ms}$ for different microchannel temperatures. Recorded voltages follow a function of $\theta^{4}$ as predicted by Equation (11). Therefore, by increasing the temperature of the device in the range of $25-65^{\circ} \mathrm{C}$, the total amount of thermal energy proportionally decreases, resulting in a lower pyroelectric charge generation. The analytical and the theoretical results are shown by comparing the pyroelectric peak response versus temperature in Figures 6 and 8 . Sensor signal coming out of the charge amplifier is opposite in sign with respect to the analytical response, primarily due to the topology of the preamplifier.

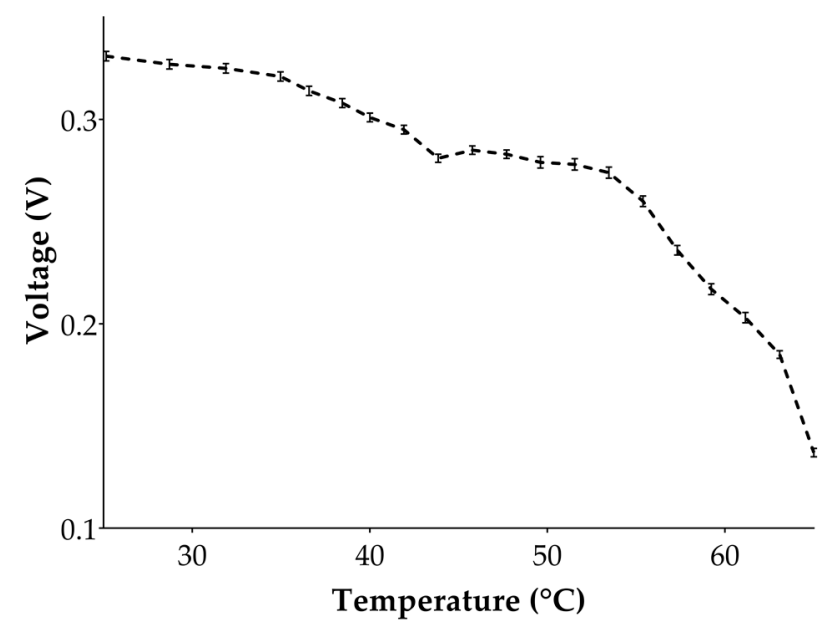

Figure 8. Calibration curve of the pyroelectric sensor performed inside the microfluidic channel in the temperature range $25-65{ }^{\circ} \mathrm{C}$ (as reported in the insert of Figure 7). Charge amplifier voltage are evaluated for each target temperature at the time $t=T_{I R}$. These data are expressed as mean $\pm \mathrm{SD}$; for each target temperature sensor output voltage was evaluated three times, as reported in the error bars.

The application of incident IR-shaped radiation on a properly designed pyroelectric transducer and a read-out circuit, combined with a heat transfer model allows measurement of the absolute temperature of an object without the use of adjunctive reference temperature sensors [18-20]. The scheme reported in this paper overcomes the problems associated with the evaluation of temperature gradient as previously reported in the literature by using a single dynamic sensor. A comparison among the proposed pyroelectric-based sensor with a selected group of sensors realized using state-of-art material technologies is reported in Table 2. It can be seen from the table that PVDF offers a faster response time even though other materials possess higher maximum working temperatures. However, the temperature range of the pyroelectric sensors is suitable for most microfluidic applications. Investigations of different pyroelectric materials such as PMN-PT crystals have been reported in the literature. These materials possess high-voltage response and potentially represent a promising sensor material. However, they are more expensive compared with PVDF and are typically fragile, which makes them less attractive for commercial applications. Furthermore, PVDF possesses a higher coupling factor than PZT and is also less expensive while demonstrating a high degree of mechanical stability and stable temperature variations. Type T Ultra-Fast Thermocouple can represent a valid counterpart in terms of dimensions and response time for microfluidic applications, although it requires direct contact with the fluid. 
Table 2. Characteristics of the proposed pyroelectric sensor compared to other sensor technologies.

\begin{tabular}{ccccc}
\hline Technology & Response Time [s] & Dimensions $[\mathbf{m m}]$ & Temp. Range $\left[{ }^{\circ} \mathbf{C}\right]$ & Reference \\
\hline PZT & 5 & $1.2 \times 10^{-3}$ & $80-110$ & {$[42]$} \\
PZT & 0.00875 & $1.6 \times 10^{-3}$ & $31-62$ & {$[43]$} \\
PZT & 0.42 & 0.15 & $14-93.5$ & {$[44]$} \\
LiTaO & 0.01 & $5.21 \times 10^{-2}$ & $27-37$ & {$[45]$} \\
PMN-30PT & 0.1 & $8.64 \times 10^{-3}$ & $32-40.4$ & {$[46]$} \\
PMN-25PT & 1 & 3.76 & $\mathrm{~N} / \mathrm{A}$ & {$[47]$} \\
PVDF & 0.0032 & 0.25 & $-40-65$ & {$[14,20]$} \\
T-type Thermoc. & 0.005 & 0.22 & $-273-150$ & {$[48]$} \\
\hline
\end{tabular}

Calibration is needed in order to obtain an accurate prediction of the peak output voltage. Close similarities between the analytical and the experimental results prove the consistency of the adopted analysis. Discrepancies can mainly be attributed to the hot chuck setup, the sensitivity of the pyroelectric material to the electromagnetic interference and also to the charge amplifier which produces noise in the output voltage resulting in lowering of the sensitivity. Being a non-linear calibration curve, accuracy is not consistent and error is higher at a lower temperature. Output voltage can be evaluated at a rate defined by the frequency band of the transducer $(0.8-49.7 \mathrm{~Hz})$, allowing the evaluation of temperature dynamic inside the microchannel characterized by inherent low thermal inertia at high frequency. Accuracy should be maintained while designing the metallized surfaces in order to fully characterize the device in terms of adsorption and irradiance.

\section{Conclusions}

The research outcomes presented in the paper address the establishment of an innovative way of using pyroelectric thin film for the fabrication of high-performance and cost-effective temperature sensors. Compared to previous works, the actual pyroelectric sensor was developed to evaluate the absolute temperature instead of temperature gradient (with no knowledge about the initial and the final temperatures without the use of additional sensors). In the actual work, the development of an alternate approach involving an IR laser and a thermal model that take into account the heat exchange mechanisms of the active element was presented. Because the pyroelectric transducers do not need to reach thermal equilibrium, there is no latency for the thermalization of the device. This allows the pyroelectric transducers to operate at higher frequencies compared to the traditional thermometers. The period of IR emission, the wavelength, and the power emitted can also be considered to be the key points for optimizing the trade-off between the sensitivity and the temperature frequency acquisition. The performance of the pyroelectric transducer can be remarkably improved and a higher level of integration can be achieved for the realization of the microsystems incorporating low-noise CMOS charge amplifier and filtering stages on a silicon platform. These factors can all play a strategic role in the fabrication of a dedicated lab-on-a-chip for biomedical application.

Acknowledgments: The authors are grateful to Prof. Philip Rack and Carlos Gonzalez of the University of Tennessee at Knoxville for their support, and to BATS S.r.l., Reggio Calabria, Italy, for the support in the production of the sensor.

Author Contributions: Salvatore A. Pullano designed and performed the experiments, analyzed the data, compiled the manuscript, and prepared the figures. Antonino S. Fiorillo and Syed K. Islam contributed to analysis of the results. Salvatore Andrea Pullano, Syed K. Islam, Ifana Mahbub, and Antonino S. Fiorillo. critically revised the article and approved the final version. Antonino S. Fiorillo supervised the overall work. The authors are accountable for all aspects of the work and ensure that questions related to the accuracy or integrity of any part of the work are appropriately investigated and resolved.

Conflicts of Interest: The authors declare no conflict of interest. 


\section{Abbreviations}

The following abbreviations are used in this manuscript:

$\begin{array}{ll}\text { SOC } & \text { System-On-a-Chip } \\ \text { PVDF } & \text { Polyvinylidene-Fluoride } \\ \text { PZT } & \text { Lead Zirconate Titanate } \\ \text { PMMA } & \text { Poly(methyl methacrylate) } \\ \text { IR } & \text { Infrared } \\ \text { LED } & \text { Light Emitting Diode } \\ \text { CMOS } & \text { Complementary Metal Oxide Semiconductor }\end{array}$

\section{References}

1. Ghallab, Y.H.; El-Hamid, H.A.; Ismail, Y. Lab on a Chip Based on CMOS Technology: System Architectures, Microfluidic Packaging, and Challenges. IEEE Des. Test 2015, 32, 20-31. [CrossRef]

2. Kubicki, W.; Walczak, R.; Dziuban, J.A. Miniature instrument for lab-on-a-chip capillary gel electrophoresis of DNA utilizing temperature control technique. In Proceedings of the Eurosensors XXV, Athens, Greece, 4-7 September 2011; pp. 1237-1240.

3. Cao, J.; Cui, F.; Chen, W.; Guo, Z.; Chen, W. Analysis on PCR chip with reusable electrodes and its temperature field. Bandaoti Guangdian Semicond. Optoelectron. 2015, 38, 725-727, 732.

4. Wu, Z.Y.; Chen, K.; Qu, B.Y.; Tian, X.X.; Wang, X.J.; Fang, F. A thermostat chip of indium tin oxide glass substrate for static polymerase chain reaction and in situ real time fluorescence monitoring. Anal. Chim. Acta 2008, 610, 89-96. [CrossRef] [PubMed]

5. Martinez-Quijada, J.; Caverhill-Godkewitsch, S.; Reynolds, M.; Gutierrez-Rivera, L.; Johnstone, R.W.; Elliott, D.G.; Sameoto, D.; Backhouse, C.J. Fabrication and characterization of aluminum thin film heaters and temperature sensors on a photopolymer for lab-on-chip systems. Sens. Actuators A Phys. 2013, 193, 170-181. [CrossRef]

6. Li, P.; Lei, N.; Sheadel, D.A.; Xu, J.; Xue, W. Integration of nanosensors into a sealed microchannel in a hybrid lab-on-a-chip device. Sens. Actuators B Chem. 2012, 166-167, 870-877. [CrossRef]

7. Kandlikar, S.G. History, advances, and challenges in liquid flow and flow boiling heat transfer in microchannels: A critical review. J. Heat Transf. 2012, 134, 3. [CrossRef]

8. Jurków, D.; Roguszczak, H.; Golonka, L. Novel cold chemical lamination bonding technique-A simple LTCC thermistor-based flow sensor. J. Ceram. Soc. 2009, 29, 1971-1976. [CrossRef]

9. Wu, T.; Liu, Y.; Yu, Z.; Ye, H.; Peng, Y.; Shu, C.; Yang, C.; Zhang, W.; He, H. A nanometeric temperature sensor based on plasmonic waveguide with an ethanol-sealed rectangular cavity. Opt. Commun. 2015, 339, 1-6. [CrossRef]

10. Donner, J.S.; Thompson, S.A.; Kreuzer, M.P.; Baffou, G.; Quidant, R. Mapping intracellular temperature using green fluorescent protein-from in vitro to in vivo. In Proceedings of the Optical Molecular Probes, Imaging and Drug Delivery, OMP 2013, Waikoloa Beach, HI, USA, 14-18 April 2013.

11. Tanimoto, R.; Hiraiwa, T.; Nakai, Y.; Shindo, Y.; Oka, K.; Hiroi, N.; Funahashi, A. Detection of Temperature Difference in Neuronal Cells. Sci. Rep. 2016, 6. [CrossRef] [PubMed]

12. Bergaud, C. Thermometry in micro and nanofluidics. In Thermometry at the Nanoscale: Techniques and Selected Applications; Carlos, L.D., Palacio, F., Eds.; Royal Society of Chemistry: London, UK, 2016; Volume 2016, pp. 461-492.

13. Puddu, M.; Mikutis, G.; Stark, W.J.; Grass, R.N. Submicrometer-Sized Thermometer Particles Exploiting Selective Nucleic Acid Stability. Small 2016, 12, 452-456. [CrossRef] [PubMed]

14. Measurement Specialties Inc. Piezo Film Sensors Technical Manual. Available online: https://www.sparkfun. com/datasheets/Sensors/Flex/MSI-techman.pdf (accessed on 31 August 2016).

15. Sarker, M.R.H.; Karim, H.; Martinez, R.; Delfin, D.; Enriquez, R.; Ishtiaque Shuvo, M.A.; Love, N.; Lin, Y. Temperature measurements using a lithium niobate (LiNbO3) pyroelectric ceramic. Measurement 2015, 75, 104-110. [CrossRef]

16. Maiolo, L.; Maita, F.; Pecora, A.; Rapisarda, M.; Mariucci, L.; Benwadih, M.; Jacob, S.; Chartier, I.; Coppard, R. Flexible PVDF-TrFE pyroelectric sensor integrated on a fully printed p-channel organic transistor. Procedia Eng. 2012, 47, 526-529. [CrossRef] 
17. Cardoso, V.F.; Correia, R.G.; Rocha, J.G.; Lanceros-Mendéz, S.; Minas, G. Design and fabrication of piezoelectric microactuators based on $\beta$-poly(vinylidene fluoride) films for microfluidic applications. In Proceedings of the 32nd Annual International Conference of the IEEE EMBS, Buenos Aires, Argentina, 31 August-4 September 2010.

18. Pullano, S.A.; Perozziello, G.; Di Fabrizio, E.; Fiorillo, A.S. Pyroelectric PVDF transducer for temperature measurements in fluidic micro-channels. In Proceedings of the E-Health and Bioengineering Conference (EHB), Iasi, Romania, 1-4 November 2011.

19. Pullano, S.A.; Fiorillo, A.S.; Islam, S.K. A pyroelectric sensor for system-on-a-chip. In Proceedings of the 40th Annual Northeast Bioengineering Conference (NEBEC), Boston, MA, USA, 1-2 April 2014.

20. Pullano, S.A.; Islam, S.K.; Fiorillo, A.S. Pyroelectric Sensor for Monitoring the Temperature of Biological Fluids in Micro Channel Devices. IEEE Sens. J. 2014, 14, 2725-2730. [CrossRef]

21. Sung, M.; Shin, K.; Moon, W. A new transduction mechanism for hydrophones employing piezoelectricity and a field-effect transistor. Sen. Actuators A Phys. 2015, 233, 557-568. [CrossRef]

22. Peng, G.; Zhao, X.; Zhan, Z.; Ci, S.; Wang, Q.; Liang, Y.; Zhao, M. New crystal structure and discharge efficiency of poly(vinylidene fluoride-hexafluoropropylene)/poly(methyl methacrylate) blend films. RCS Adv. 2014, 4, 16849-16854. [CrossRef]

23. Beuville, E.; Buytaert, S.; Cerri, C.; Jarron, P. Low Noise Analog CMOS Signal Processor with a Large Dynamic Range for Silicon Calorimeters. Nucl. Phys. B Proc. Suppl. 1991, 23, 198-206. [CrossRef]

24. Junnila, S.; Akbhardeh, A.; Varri, A. An Electromechanical Film Sensor Based Wireless Ballistocardiographic Chair: Implementation and Performance. J. Signal Proc. Syst. 2009, 57, 305-320. [CrossRef]

25. Sultana, A.; Reznik, A.; Karim, K.S.; Rowlands, J.A. Design and Feasibility of Active Matrix Flat Panel Detector Using Avalanche Amorphous Selenium for Protein Crystallography. Med. Phys. 2008, 35, 4324-4332. [CrossRef] [PubMed]

26. Maji, S.; Sarkar, P.K.; Aggarwal, L.; Ghosh, S.K.; Mandal, D.; Sheet, G.; Acharya, S. Self-oriented $\beta$-crystalline phase in the polyvinylidene fluoride ferroelectric and piezo-sensitive ultrathin Langmuir-Schaefer film. Phys. Chem. Chem. Phys. 2015, 17, 8159-8165. [CrossRef] [PubMed]

27. Tseng, H.-J.; Tian, W.-C.; Wu, W.-J. Flexible PZT Thin Film Tactile Sensor for Biomedical Monitoring. Sensors 2013, 13, 5478-5492. [CrossRef] [PubMed]

28. Zammit, U.; Marinelli, M.; Mercuri, F.; Paoloni, S.; Scudieri, F. Photopyroelectric calorimeter for the simultaneous thermal, optical, and structural characterization of samples over phase transitions. Rev. Sci. Instrum. 2011, 82, 12. [CrossRef] [PubMed]

29. Lang, S.B. Pyroelectricity: Form ancient curiosity to modern imaging tool. Phys. Today 2005, 58, 8. [CrossRef]

30. Touayar, O.; Sifi, N.; Ben Brahim, J. Theoretical and experimental study of power radiometric measurements using a pyroelectric current integrator converter. Sens. Actuators A Phys. 2006, 135, 484-491. [CrossRef]

31. Shao, X.; Ding, J.; Ma, X.; Yu, Y.; Fang, J. Document Design and thermal analysis of electrically calibrated pyroelectric detector. Infrared Phys. Technol. 2012, 55, 45-48. [CrossRef]

32. Chirtoc, M.; Bentefour, E.H.; Antoniow, J.S.; Glorieux, C.; Thoen, J.; Delenclos, S.; Sahraoui, A.H.; Longuemart, S.; Kolinsky, C.; Buisine, J.M. Current mode versus voltage mode measurement of signals from pyroelectric sensors. Rev. Sci. Instrum. 2003, 74, 648-650. [CrossRef]

33. Gilber, B. Current Mode, Voltage Mode, or Free Mode? A Few Sage Suggestion. Analog Integr. Circuits Signal Proc. 2004, 38, 83-101. [CrossRef]

34. Kester, W.; Bryant, J.; Jung, W.; Wurcer, S.; Kitchin, C. Sensor Signal Conditioning. In Op Amp Applications Handbook; Jung, W.G., Ed.; Analog Device Series: Norwood, MA, USA, 2004.

35. López-Martin, A.J.; Massarotto, M.; Carlosena, A. Performance tradeoffs of integrated CMOS charge amplifiers. In Proceedings of the IEEE Sensors 2009 Conference, Christchurch, New Zealand, 25-28 October 2009.

36. Lubomirsky, I.; Stafsudd, O. Invited Review Article: Practical guide for pyroelectric measurements. Rev. Sci. Instrum. 2012, 83, 051101. [CrossRef] [PubMed]

37. Tsai, C.F.; Young, M.S. Pyroelectric infrared sensor-based thermometer for monitoring indoor objects. Rev. Sci. Instrum. 2003, 74, 12. [CrossRef]

38. Yoon, H.W.; Eppeldauer, G.P. Radiation Thermometer Designs, in: Radiometric Temperature Measurements. Exp. Methods Phys. Sci. 2009, 42, 133-180.

39. Querner, Y.; Norkus, V.; Gerlach, G. High-sensitive Pyroelectric detectors with internal thermal amplification. Sens. Actuators A Phys. 2011, 172, 169-174. [CrossRef] 
40. Campanella, H.; Narducci, M.; Soon, J.B.W.; Merugu, S.; Ferrari, M.; Ferrari, V.; Singh, N. Multi-sensitive temperature sensor platforms using aluminum nitride MEMS resonators. J. Micromech. Microeng. 2015, 25, 1-12. [CrossRef]

41. DeWitt, D.P.; Nutter, G.D. Theory and Practice of Radiation Thermometry; Wiley-Interscience: New York, NY, USA, 1988.

42. Buchanan, R.; Huang, J. Pyroelectric and sensor properties of ferroelectric thin films for energy conversion. J. Eur. Ceram. Soc. 1999, 19, 1467-1471. [CrossRef]

43. Cuadras, M.; Gasulla Ferrari, V. Thermal energy harvesting through pyroelectricity. Sens. Actuators A Phys. 2010, 158, 132-139. [CrossRef]

44. Ravindran, S.K.T.; Huesgen, T.; Kroener, M.; Woias, P. A Self-Sustaining Pyroelectric Energy Harvester Utilizing Spatial Thermal Gradients. In Proceedings of the 16th International Solid-State Sensors, Actuators and Microsystems Conference, TRANSDUCERS'11, Beijing, China, 5-9 June 2011.

45. Chang, H.H.S.; Huang, Z. Laminate composites with enhanced pyroelectric effects for energy harvesting. Smart Mater. Struct. 2010, 19, 1. [CrossRef]

46. Mane, P.; Xie, J.; Leang, K.; Mossi, K. Cyclic energy harvesting from pyroelectric materials. IEEE Trans. Ultrason. Ferroelectr. Freq. Control 2011, 58, 10-17. [CrossRef] [PubMed]

47. Sebald, G.; Lefeuvre, E.; Guyomar, D. Pyroelectric energy conversion: Optimization principles. IEEE Trans. Ultrason. Ferroelectr. Freq. Control 2008, 55, 538-551. [CrossRef] [PubMed]

48. ADInstruments. Available online: http://m-cdn.adinstruments.com/product-data-cards/MLT1402-DCW15A.pdf (accessed on 16 May 2016).

(C) 2017 by the authors. Licensee MDPI, Basel, Switzerland. This article is an open access article distributed under the terms and conditions of the Creative Commons Attribution (CC BY) license (http:/ / creativecommons.org/licenses/by/4.0/). 